\title{
Retrospective comparative study of IV+ local tranexamic acid versus IV tranexamic acid only in primary total knee replacement
}

\author{
Sanil G. Kamat ${ }^{1 *}$, Rohan Dessai ${ }^{2}$ \\ ${ }^{1}$ Department of Orthopaedics, The Princess Alexandra Hospital NHS trust, United Kingdom \\ ${ }^{2}$ Department of Orthopaedics, Manipal Hospital, Goa, India
}

Received: 22 November 2020

Revised: 05 January 2021

Accepted: 06 January 2021

*Correspondence:

Dr. Sanil G Kamat,

E-mail: kamat.sanil@gmail.com

Copyright: ( $)$ the author(s), publisher and licensee Medip Academy. This is an open-access article distributed under the terms of the Creative Commons Attribution Non-Commercial License, which permits unrestricted non-commercial use, distribution, and reproduction in any medium, provided the original work is properly cited.

\begin{abstract}
Background: The study is to compare the immediate post-operative outcomes with use of intravenous (IV) tranexamic acid (TXA) versus IV and local TXA combination in primary unilateral total knee arthroplasty. Study comprised of 72 cases of tricompartmental knee primary osteoarthritis who have undergone unilateral total knee arthroplasty at Manipal Hospital, Goa from January 2016 to December 2018. The observations for each group was analysed and post op blood loss in drain, fall of haemoglobin levels and need of blood transfusion was recorded. The results were statistically compared. The mean blood loss fall in HB levels and need of blood transfusions revealed statistically significant differences.

Methods: Total 72 patients diagnosed with primary tricompartmental osteoarthritis were divided into two groups retrospectively. Group 1 (IV only): $1 \mathrm{gm}$ IV Tranexamic acid bolus $10 \mathrm{~min}$ before deflating the tourniquet. Group 2 (IV + Local): $1 \mathrm{gm}$ IV Tranexamic acid bolus $10 \mathrm{~min}$ before deflating the tourniquet and $1 \mathrm{gm}$ Tranexamic Acid in $50 \mathrm{ml}$ saline locally at the time of closure.

Results: It was observed that higher post op blood loss, higher fall in haemoglobin (HB) levels and higher requirement of blood transfusions were associated with group 1 as compared to 2 .

Conclusions: The study inferred that the combination of local and systemic tranexamic acid was superior than systemic administration alone with lower post op blood loss, lower rates of blood transfusion and lower fall in haemoglobin levels without any added complications.
\end{abstract}

Keywords: Total knee replacement, Tranexamic acid, Haemoglobin, Blood transfusion

\section{INTRODUCTION}

Total knee replacement is the only surgical option currently available in case of well-established tricompartmental osteoarthritis. Intra and postoperative bleeding remains one of the main concerns in total knee arthroplasty (TKA), with blood loss ranging between $1000 \pm 500 \mathrm{ml} .{ }^{1}$ The use of a pneumatic tourniquet ensures a dry surgical field and minimal intraoperative bleeding, but it augments fibrinolysis stimulated by surgical trauma. ${ }^{2-7}$ This activation of the fibrinolytic system might lead to high postoperative blood loss . Different methods to reduce perioperative blood loss have been studied, such as perioperative blood donation, perioperative red cell salvage, deliberate hypotension, and use of recombinant human erythropoietin. Furthermore, perioperative transfusions add to the costs of the treatment and the risks (of infection, allergic reaction and disease transmission, for example) to the patient. ${ }^{8}$

In recent times, pharmacological approaches are attracting a lot of attention. As hyperfibrinolysis is considered one of 
the major causes of postoperative bleeding after TKA surgery, antifibrinolytic drugs have been proposed, including aprotin, aminocaproic acid, and tranexamic acid (TXA).

\section{Tranexamic acid a-12 $^{9-1}$}

Tranexamic acid is used in orthopaedic surgery to reduce blood loss, to the extent of reducing or altogether abolishing the need for perioperative blood collection. It is of proven value in clearing the field of surgery and reducing blood loss when given before or after surgery. Drain and number of transfusions are reduced.

Tranexamic acid is a synthetic analogy of the amino acid lysine. It serves as an antifibrinolytic by reversibly binding four to five lysine receptor sites on plasminogen. This reduces conversion of plasminogen to plasmin, preventing fibrin degradation and preserving the framework of fibrin's matrix structure. Tranexamic acid has roughly eight times the antifibrinolytic activity of an older analogue, $\varepsilon$-aminocaproic acid. TXA inhibits fibrin cleavage, thus reduc-ing the risk of haemorrhage. TXA also directly inhibits the activity of plasmin with weak potency $(\mathrm{IC} 50=87 \mathrm{mM})$ and it can block the active-site of urokinase plasminogen activator (uPA) with high specificity $(\mathrm{Ki}=2 \mathrm{mM})$ among all the serine proteases. Plasma protein binding of 3\%. Low hepatic metabolism and $95 \%$ renal clearance and half-life approximately 2.3 hours.

The aim of the present study was to compare the efficacy of systemic versus systemic plus local administration of TXA in reducing blood loss after TKA.

\section{METHODS}

\section{Source of data}

The retrospective study was conducted in a tertiary institute by collecting data of 72 cases of tricompartmental knee primary osteoarthritis who have undergone unilateral total knee arthroplasty at Manipal Hospital, Goa from January 2016 to December 2018.

The data collected from medical record department of Manipal Hospital. Data was statistically analysed to reach a conclusion. Analysis is descriptive with limitation as patients were analysed from the time of surgery till the post operative day 3. All cases operated by two surgeons with same techniques and same anaesthesia protocol.

\section{Study design}

This is a comparative retrospective study in which patients were admitted and operated at Manipal Hospital from January 2016 to December 2018. The comparison in terms of: post-operative blood loss, fall in haemoglobin level post operative, post operative blood transfusion rate.
All patients underwent TKR by cemented, medial parapatellar, and posterior cruciate ligament substituting method. Tourniquet were used in all patient which was inflated before the incision and was deflated at the time of closure. Tablet Rivaroxaban $10 \mathrm{mg}$ OD started for all patients from POD1 for prophylaxis against DVT. All patients received rehabilitation after the surgery including pain management, exercises for Range of Motion (ROM) re-gaining and muscular strength enhancement. The exercises majorly included active ankle pumping for deep venous thrombosis prevention and oedema control, active and active-assisted ROM exercises of knee flexion and extension, strengthening knee joint musculature with special emphasis on knee extensors by isometric method progressing to isotonic exercise and gait education with walker and cane. Exercise performance was supervised by a physiotherapist.

\section{Ethical approval}

No ethical approval was required as it's a retrospective study and patients personal details not mentioned in the study.

Software statistical package for social sciences (SPSS) version 20.0 used for statistical analysis.

\section{Use of tranexamic acid}

Group 1 (IV only)

$1 \mathrm{gm}$ IV Tranexamic acid bolus $10 \mathrm{~min}$ before deflating the tourniquet.

Group $2(I V+$ Local $)$

$1 \mathrm{gm}$ IV Tranexamic acid bolus $10 \mathrm{~min}$ before deflating the tourniquet and $1 \mathrm{gm}$ Tranexamic Acid in $50 \mathrm{ml}$ saline locally at the time of closure.

\section{Inclusion criteria}

Surgically fit patients more than 50 years of age who has been diagnosed as having tri compartmental primary osteoarthritis knee.

\section{Exclusion criteria}

Known RA of knee, complicated surgeries with need for computer navigation, age less than 50 years, patients with preop $\mathrm{Hb}$ levels less than 10, revision TKRs, patients with contraindications to Tranexamic acid administration (a medical history of DVT or pulmonary thromboembolism, acute myocardial infarction, heart failure, heart valve stenosis, ischemic stroke, coagulopathy, allergy to TXA, severe liver or kidney disease), patient with known coagulation disorders. 


\section{RESULTS}

The average post operative drain on day 1 in group 1 cases was 275.4 which was noted to be significantly higher than in group 2.

Table 1: Post-operative day1 drain collection.

\begin{tabular}{|lllll|}
\hline Group statistics (significant) & & \\
\hline & $\begin{array}{l}\text { Tranexamic } \\
\text { acid }\end{array}$ & N & Mean & $\begin{array}{l}\text { Std. } \\
\text { Deviation }\end{array}$ \\
\hline $\begin{array}{l}\text { Post op } \\
\text { D1 drain } \\
\text { (in ml) }\end{array}$ & Group1 & 35 & 275.43 & 92.192 \\
\cline { 2 - 5 } & Group2 & 37 & 179.57 & 114.250 \\
\hline
\end{tabular}

Furthermore, the average post op drain on day 2 in group 1 also appeared to be considerably higher than group 2 by almost $40.6 \mathrm{ml}$. In Addition to this, more number of drains were retained in group 1 patients as compared to group 2 on post operative day.

Table 2: Post-operative day2 drain collection.

\begin{tabular}{|lllll|}
\hline Group statistics (highly significant) & \\
& $\begin{array}{l}\text { Tranexamic } \\
\text { acid }\end{array}$ & N & Mean & $\begin{array}{l}\text { Std. } \\
\text { Deviation }\end{array}$ \\
\hline $\begin{array}{l}\text { Post op } \\
\text { D2 drain } \\
\text { (in ml) }\end{array}$ & Group1 & 35 & 128.66 & 44.881 \\
\cline { 2 - 5 } & Group2 & 37 & 88.24 & 58.360 \\
\hline
\end{tabular}

Also, preoperative hemoglobin levels were almost identical in both the groups, however there was a significant drop in post op hemoglobin in group 1 as compared to group 2. Lastly, higher incidences of blood transfusion was observed in group 1 but it was not statistically significant.

Table 3: Day 3 post-operative drain retention.

\begin{tabular}{|c|c|c|c|c|c|}
\hline & & & \multicolumn{2}{|c|}{ Post op D3 drain } & \multirow{2}{*}{ Total } \\
\hline & & & Retained & Removed & \\
\hline \multirow{4}{*}{$\begin{array}{l}\text { Local } \\
\text { Tranexamic acid }\end{array}$} & \multirow{2}{*}{ Group1 } & Count & 12 & 23 & 35 \\
\hline & & $\%$ within Local Tranexamic acid & $34.3 \%$ & $65.7 \%$ & $100.0 \%$ \\
\hline & \multirow{2}{*}{ Group2 } & Count & 0 & 37 & 37 \\
\hline & & $\%$ within Local Tranexamic acid & $0.0 \%$ & $100.0 \%$ & $100.0 \%$ \\
\hline \multirow{2}{*}{ Total } & & Count & 12 & 60 & 72 \\
\hline & & $\%$ within Local Tranexamic acid & $16.7 \%$ & $83.3 \%$ & $100.0 \%$ \\
\hline
\end{tabular}

Table 4: Preoperative hemoglobin levels.

\begin{tabular}{|c|c|c|c|c|}
\hline \multicolumn{5}{|c|}{ Group Statistics (Not Significant) } \\
\hline & Local tranexamic acid & $\mathbf{N}$ & Mean & Std. Deviation \\
\hline \multirow{2}{*}{$\begin{array}{l}\text { Pre-operative Hb (in } \\
\text { gm/dl) }\end{array}$} & Group1 & 35 & 12.78429 & 1.103042 \\
\hline & Group2 & 37 & 12.62173 & 1.060483 \\
\hline
\end{tabular}

Table 5: Post-operative hemoglobin levels.

\begin{tabular}{|lllll|}
\hline Group Statistics (Not Significant) & Local tranexamic acid & N & Mean & Std. Deviation \\
\hline & Group1 & 35 & 11.11200000000 & 1.121652667220273 \\
\multirow{2}{*}{$\begin{array}{l}\text { Post-operative Hb } \\
\text { (in gm/dl) }\end{array}$} & Group2 & 37 & $\begin{array}{l}11.48445945945 \\
9460\end{array}$ & 1.258539598869230 \\
\cline { 2 - 5 } & & 37 & 94600 \\
\hline
\end{tabular}

Table 6: Fall in hemoglobin levels.

\begin{tabular}{|llllll|}
\hline \multicolumn{2}{|c|}{ Group Statistics (Very Highly Significant) } & & & & \\
\hline \multirow{2}{*}{ Fall in Hb } & Local Tranexamic acid & N & Mean & Std. Deviation & Std. Error Mean \\
\cline { 2 - 6 } & Group1 & 35 & 1.6780 & 0.63172 & 0.10678 \\
\hline
\end{tabular}


Table 7: Blood transfusions.

Local Tranexamic acid * Packed red blood cell Crosstabulation (Not Significant)

\begin{tabular}{|c|c|c|c|c|c|}
\hline & & & \multicolumn{2}{|c|}{ Packed red blood cell } & \multirow{2}{*}{ Total } \\
\hline & & & 1 & Not given & \\
\hline \multirow{4}{*}{$\begin{array}{l}\text { Local Tranexamic } \\
\text { acid }\end{array}$} & \multirow[b]{2}{*}{ Group1 } & Count & 4 & 31 & 35 \\
\hline & & $\begin{array}{l}\% \text { within Local Tranexamic } \\
\text { acid }\end{array}$ & $11.4 \%$ & $88.6 \%$ & $100.0 \%$ \\
\hline & \multirow[b]{2}{*}{ Group2 } & Count & 1 & 36 & 37 \\
\hline & & $\begin{array}{l}\% \text { within Local Tranexamic } \\
\text { acid }\end{array}$ & $2.7 \%$ & $97.3 \%$ & $100.0 \%$ \\
\hline \multirow[b]{2}{*}{ Total } & & Count & 5 & 67 & 72 \\
\hline & & $\begin{array}{l}\% \text { within Local Tranexamic } \\
\text { acid }\end{array}$ & $6.9 \%$ & $93.1 \%$ & $100.0 \%$ \\
\hline
\end{tabular}

\section{DISCUSSION}

Perioperative blood loss is an inevitable complication of revision TKA, which could lead to anaemia. Effective blood management can minimize blood loss and transfusions such that patients achieve better results. This study demonstrated that TXA is effective in reducing total blood loss, transfusion rate and blood in the drain, without increasing the rate of complications. Furthermore far better results in terms of lesser post-operative blood loss, lesser rates of blood transfusions and lower fall of post operative $\mathrm{Hb}$ was evident when Systemic Tranexamic acid was coupled with local infusion.

Table 8: Summary of meta-analyses on the efficacy of tranexamic acid in total knee arthroplasty.

\begin{tabular}{|c|c|c|c|c|c|}
\hline Author & Year & Protocol & Studies & $\begin{array}{l}\text { Outcomes } \\
\text { evaluated }\end{array}$ & Results \\
\hline $\begin{array}{l}\text { Alshryda } \\
\text { et } \text { al }^{17}\end{array}$ & 2011 & $\begin{array}{l}\text { IV or } \\
\text { topical or } \\
\text { oral }\end{array}$ & $\begin{array}{l}19 \text { RCTs (18 } \\
\text { involving IV } \\
\text { administration, } \\
1 \text { IA } \\
\text { administration, } \\
\text { and } 1 \text { oral } \\
\text { administration) }\end{array}$ & $\begin{array}{l}\text { Blood loss, } \\
\text { transfusion rate }\end{array}$ & $\begin{array}{l}\text { Reduction in blood loss (MD } 591 \mathrm{ml} ; 95 \% \text { CI } \\
536 \text { to } 647, \mathrm{p}<0.001 \text {; heterogeneity } \mathrm{I}^{2}=78 \% \text { ), } \\
\text { reduction in transfusion rate (RR } 2.56,95 \% \text { CI } \\
2.1 \text { to } 3.1, \mathrm{p}<0.001 \text {; heterogeneity } \mathrm{I}^{2}=75 \% \text { ) }\end{array}$ \\
\hline $\begin{array}{l}\text { Zhang et } \\
\text { al }^{18}\end{array}$ & 2012 & IV & 15 RCTs & $\begin{array}{l}\text { Blood loss, } \\
\text { transfusion rate }\end{array}$ & $\begin{array}{l}\text { Reduction in blood loss (MD } 487 \mathrm{ml}, 95 \% \text { CI } \\
-629 \text { to }-344 \text { ), reduction in transfusion rate } \\
\text { (risk difference }-0.4, p<0.00001 \text { ) }\end{array}$ \\
\hline $\begin{array}{l}\text { Panteli et } \\
\text { al }^{19}\end{array}$ & 2013 & Topical & $\begin{array}{l}7 \text { ( } 4 \text { RCTs, } 1 \\
\text { PCS, } 2 \text { quasi- } \\
\text { RCTs) }\end{array}$ & $\begin{array}{l}\text { Blood loss, } \\
\text { haemoglobin } \\
\text { drop, transfusion } \\
\text { rate }\end{array}$ & $\begin{array}{l}\text { Reduction in total blood loss }(\mathrm{MD}=-220.08 \\
\mathrm{ml}, \mathrm{p}<0.00001,95 \% \mathrm{CI}=-279.54 \mathrm{ml} \text { to } \\
-160.63 \mathrm{ml}), \text { maximum haemoglobin drop } \\
(\mathrm{MD}=-0.94 \mathrm{gr} / \mathrm{dl}, 95 \% \mathrm{CI}=-1.24 \mathrm{gr} / \mathrm{dl} \text { to } \\
-0.65 \mathrm{gr} / \mathrm{dl}, \mathrm{p}<0.00001) ; \text { lower risk of } \\
\text { transfusions }(\mathrm{RR}=0.47, \mathrm{p}=0.01,95 \% \mathrm{CI}= \\
0.26 \text { to } 0.84)\end{array}$ \\
\hline $\begin{array}{l}\text { Wang et } \\
\text { al20 }\end{array}$ & 2014 & $\begin{array}{l}\text { IV or } \\
\text { topical }\end{array}$ & $\begin{array}{l}6(5 \text { RCTs, } 1 \\
\text { PCS })\end{array}$ & $\begin{array}{l}\text { Total blood loss, } \\
\text { Hemoglobin } \\
\text { drop, drain } \\
\text { output, } \\
\text { transfusion rate }\end{array}$ & $\begin{array}{l}\text { No differences as regards total blood loss (MD } \\
-14.36,95 \% \text { CI }-92.02 \text { to } 63.30) \text {, } \\
\text { haemoglobin loss (MD } 0.43,95 \% \text { CI }-0.25 \text { to } \\
1,11 \text { ), total drain output (MD } 21.91,95 \% \text { CI } \\
-85.01 \text { to } 128.82 \text { ), transfusion rate (RR } 1.02 \text {, } \\
95 \% \text { CI } 0.70 \text { to } 1.9 \text { ) }\end{array}$ \\
\hline $\begin{array}{l}\text { Alshryda } \\
\text { et } \mathbf{a l}^{21}\end{array}$ & 2014 & Topical & $\begin{array}{l}14 \text { RCTs }(11 \\
\text { knee } \\
\text { replacement, } 1 \\
\text { hip } \\
\text { replacement, } 1 \\
\text { both) }\end{array}$ & Transfusion rate & $\begin{array}{l}\text { Reduction in transfusion rate }(\mathrm{RR} 4.51 ; 95 \% \\
\text { CI: } 3.02 \text { to } 6.72 ; \mathrm{p}<0.001 \text {; heterogeneity } \mathrm{I}^{2}= \\
0 \%)\end{array}$ \\
\hline
\end{tabular}




\begin{tabular}{|c|c|c|c|c|c|}
\hline Author & Year & Protocol & Studies & $\begin{array}{l}\text { Outcomes } \\
\text { evaluated }\end{array}$ & Results \\
\hline $\begin{array}{l}\text { Kim et } \\
\text { al }^{22}\end{array}$ & 2014 & $\begin{array}{l}\text { IV or } \\
\text { topical }\end{array}$ & $\begin{array}{l}28 \text { RCTs ( } 22 \\
\text { involving IV } \\
\text { administration; } \\
6 \text { IA } \\
\text { administration) }\end{array}$ & $\begin{array}{l}\text { Blood loss, drain } \\
\text { output, } \\
\text { transfusion rate }\end{array}$ & $\begin{array}{l}\text { Reduction in blood loss (range }=191 \mathrm{ml} \text { to } \\
942 \mathrm{ml}, 14 \% \text { to } 64 \% \text { reduction), drain output } \\
\text { (range }=65 \mathrm{ml}, 8 \% \text { reduction to } 785 \mathrm{ml}, 66 \% \\
\text { reduction), haemoglobin drop (range }=0.4 \\
\mathrm{~g} / \mathrm{dl} \text {, to } 2.8 \mathrm{~g} / \mathrm{dl}, 12 \% \text { to } 70 \% \text { reduction). } \\
\text { Variability in transfusion rate and conflicting } \\
\text { results in comparison between IV and topical }\end{array}$ \\
\hline Wu et $\mathbf{a l}^{23}$ & 2015 & $\begin{array}{l}\text { IV or } \\
\text { topical }\end{array}$ & 34 RCTs & $\begin{array}{l}\text { Blood loss (intra } \\
\text { operative and } \\
\text { postoperative), } \\
\text { haemoglobin } \\
\text { drop, safety }\end{array}$ & $\begin{array}{l}\text { Reduction in total blood loss [IA }(\mathrm{SMD}-0.86 \text {, } \\
95 \% \mathrm{CI}-1.14 \text { to }-0.59, \mathrm{p}=0.000) \mathrm{IV}(\mathrm{SMD}= \\
-1.01,95 \% \mathrm{CI}-1.43 \text { to }-0.60 ; \mathrm{p}=0.00)], \text { in } \\
\text { postoperative blood loss [IA (SMD }=-1.32 \text {, } \\
95 \% \mathrm{CI}-2.08 \text { to }-0.55 ; \mathrm{p}=0.001) \text { IV }(\mathrm{SMD}= \\
-1.11,95 \% \mathrm{CI}-1.61 \text { to }-0.61 ; \mathrm{p}=0.000)] . \text { No } \\
\text { reduction in intraoperative blood loss. } \\
\text { Reduction in haemoglobin drop [IA }(\mathrm{SMD}= \\
-0.65,95 \% \mathrm{CI}-0.96 \text { to }-0.35 ; \mathrm{p}=0.000) \mathrm{IV} \\
(\mathrm{SMD}=-0.85,95 \mathrm{CI}-1.26 \text { to }-0.44 ; \\
\mathrm{p}=0.000)] . \text { No complications }\end{array}$ \\
\hline $\begin{array}{l}\text { Shemsha } \\
\text { ki et } \mathbf{a l}^{24}\end{array}$ & 2015 & $\begin{array}{l}\text { IV or } \\
\text { topical }\end{array}$ & 31 RCTs & $\begin{array}{l}\text { Blood loss, } \\
\text { transfusion rate, } \\
\text { comparison } \\
\text { between IA and } \\
\text { IV }\end{array}$ & $\begin{array}{l}\text { Reduction in blood loss [IV }(\mathrm{MD}=392.72 \mathrm{ml} \text {, } \\
95 \% \text { CI } 528.12 \text { to }-257.33 ; \mathrm{p}<0.001) \text { IA }(\mathrm{MD} \\
=282.44 \mathrm{ml}, 95 \% \mathrm{CI} 574.73 \text { to } 9.85 ; \\
\text { p }<0.001)] \text {, reduction in transfusion rate [IV } \\
\text { (RR 0.44; } 95 \% \text { CI } 0.33 \text { to } 0.59 ; \mathrm{p}<0.001) \text { IA } \\
\text { (RR } 0.27 ; 95 \% \text { CI } 0.16-0.45 ; \mathrm{p}<0.001)] \text {. No } \\
\text { differences between IV or IA administration } \\
\text { (blood loss } \mathrm{p}=0.50 ; \text { transfusion rate } \mathrm{p}=0.30)\end{array}$ \\
\hline $\begin{array}{l}\text { Yue et } \\
\text { al }^{25}\end{array}$ & 2015 & Topical & 12 RCTs & $\begin{array}{l}\text { Blood loss, } \\
\text { transfusion rate, } \\
\text { comparison of } \\
\text { dosages }\end{array}$ & $\begin{array}{l}\text { Reduction in blood loss }(\mathrm{MD}-280.65 \mathrm{ml} \text {, } \\
95 \% \mathrm{CI},-376.43 \text { to }-184.88 ; \mathrm{p}<0.00001) \text {; } \\
\text { reduction in transfusion rate (risk ratio= }=0.26 \text {; } \\
95 \% \mathrm{CI}, 0.19 \text { to } 0.37 ; \mathrm{p}<0.00001 \text {; } \\
\text { heterogeneity } \mathrm{I}^{2}=34 \% \text { ); reduction in drainage } \\
\text { output (MD }-194.59 \mathrm{ml}, 95 \% \mathrm{CI},-315.86 \text { to } \\
-73.32 ; \mathrm{p}<0.002 ; \text { heterogeneity } \mathrm{I}^{2}=63 \% \text { ). High } \\
\text { concentration is better than low concentration } \\
\text { (total blood loss: mean reduction of } 335.79 \mathrm{ml} \\
\text { in high concentration group versus } 213.47 \mathrm{ml} \\
\text { in low concentration group; transfusion rate: } \\
\text { risk ratio=0.23 in high concentration } \\
\text { group versus } 0,37 \text { in low concentration group) }\end{array}$ \\
\hline
\end{tabular}

Abbreviations: TXA = tranexamic acid; IV = intravenous; IA = intra-articular; $\mathrm{CI}=$ confidence interval; $\mathrm{MD}=$ mean difference; $\mathrm{SMD}=$ standardized mean difference; $\mathrm{RR}=$ risk ratio; $\mathrm{RCT}$ = randomized controlled trial; Quasi-RCT = quasi-randomized controlled trial; PCS $=$ prospective cohort study.

The regimen proposed in the study was based on review of literature and our experience.

It has been demonstrated via various study that TXA is effective as well as safe when given by systemic or local route. ${ }^{13}$

Levine et al., in a randomized controlled trial, demonstrated that a standard dose of $1 \mathrm{~g}$ IV can be used with the same efficacy as weighted doses $(20 \mathrm{mg} / \mathrm{kg}) .{ }^{14}$ Iwai et al. demonstrated that a double IV dose of TXA produced a further reduction of postoperative blood loss in TKA compared to a single administration, especially if the doses were given preoperatively and intraoperatively. ${ }^{15}$ Similarly, Maniar et al., also in a Prospective study (RCT), demonstrated that a three-dose regimen (adding a postoperative dose) may be even more effective than single dose regimens. ${ }^{16}$

Most of the studies summarised below confirmed the effectiveness of varied doses of IV TXA in minimising post op blood loss and need for blood transfusions. 
Table 9: Summary of randomized controlled trials on the efficacy of tranexamic acid in total knee arthroplasty.

\begin{tabular}{|c|c|c|c|c|c|}
\hline Author & Year & $\begin{array}{l}\text { Number } \\
\text { of } \\
\text { patients }\end{array}$ & Protocol & $\begin{array}{l}\text { Outcomes } \\
\text { evaluated }\end{array}$ & Results \\
\hline Jain et $\mathbf{a l}^{26}$ & 2016 & 119 & IV versuscombined & $\begin{array}{l}\text { Blood loss, } \\
\text { transfusion rate }\end{array}$ & $\begin{array}{l}\text { Combined use of IV and topical } \\
\text { TXA provided better results } \\
\text { than IV use alone: less total } \\
\text { blood loss }(p=0.001), \text { lower } \\
\text { transfusion rate }(p=0.001) \text {, } \\
\text { smaller haemoglobin drop } \\
(p=0.001) \text {. }\end{array}$ \\
\hline $\begin{array}{l}\text { Aguilera et } \\
\text { al }^{27}\end{array}$ & 2015 & 150 & $\begin{array}{l}\text { IV }(2 \mathrm{~g}) \text { or topical } \\
(1 \mathrm{~g}) \text { versus placebo }\end{array}$ & $\begin{array}{l}\text { Blood loss, } \\
\text { transfusion rate, } \\
\text { hidden blood loss, } \\
\text { safety }\end{array}$ & $\begin{array}{l}\text { Both effective in reducing loss } \\
(\mathrm{p}=0.001), \text { reduction in } \\
\text { transfusion rate; no differences } \\
\text { between the two TXA } \\
\text { groups }(\mathrm{p}=0.073)\end{array}$ \\
\hline Shen et al ${ }^{28}$ & 2015 & 92 & $\begin{array}{l}\text { IV } 15 \mathrm{mg} / \mathrm{kg} \text { in } 100 \\
\mathrm{ml} \text { saline, } 10 \\
\text { minutes before } \\
\text { tourniquet release }\end{array}$ & $\begin{array}{l}\text { Intraoperative blood } \\
\text { loss; postoperative } \\
\text { drainage at } 12 \mathrm{~h} ; \\
\text { total drain amount; } \\
\text { hidden blood loss; } \\
\text { total blood loss; } \\
\text { transfusion volumes; } \\
\text { number of } \\
\text { transfusions; } \\
\text { postoperative } \\
\text { haemoglobin at } 1,3 \text {, } \\
\text { and } 5 \text { days; D-dimer; } \\
\text { number of lower } \\
\text { limb ecchymose }\end{array}$ & $\begin{array}{l}\text { Significant reduction in } \\
\text { postoperative drainage amount } \\
\text { at } 12 \mathrm{~h}(\mathrm{p}=0.000) \text {, total drain } \\
\text { amount }(\mathrm{p}=0.000) \text {, hidden blood } \\
\text { loss }(\mathrm{p}=0.001) \text {, total blood loss } \\
(\mathrm{p}=0.004) \text {, and postoperative } \mathrm{D}- \\
\text { dimer value at } 24 \mathrm{~h}(\mathrm{p}=0.000) \text {. }\end{array}$ \\
\hline $\begin{array}{l}\text { Hourlier et } \\
\text { al }^{29}\end{array}$ & 2015 & 106 & $\begin{array}{l}\text { IV single dose } 30 \\
\mathrm{mg} / \mathrm{kg} \\
\text { intraoperative versu } \\
\mathrm{s} 10 \mathrm{mg} / \mathrm{kg}+2 \\
\mathrm{mg} / \mathrm{kg} \text { as } \\
\text { continuous infusion } \\
2 \mathrm{~h} \text { later for } 20 \mathrm{~h}\end{array}$ & Blood loss, safety & $\begin{array}{l}\text { A single bolus of TXA } 30 \\
\mathrm{mg} / \mathrm{kg} \text { is as effective as a } \\
\text { continuous infusion }(\mathrm{p}=0.68)\end{array}$ \\
\hline $\begin{array}{l}\text { Karaaslan et } \\
\text { al }^{30}\end{array}$ & 2015 & $\begin{array}{l}81 \\
\text { undergoin } \\
\text { g bilateral } \\
\text { TKA }\end{array}$ & $\begin{array}{l}\text { IV } 15 \mathrm{mg} / \mathrm{kg} 10 \\
\text { min before inflation } \\
\text { of the tourniquet } \\
\text { (and continued at } \\
10 \mathrm{mg} / \mathrm{kg} \text { for } 3 \mathrm{~h})+ \\
3 \mathrm{~g} \mathrm{IA} 10 \mathrm{~min} \\
\text { before deflation of } \\
\text { the tourniquet }\end{array}$ & $\begin{array}{l}\text { Volume of drained } \\
\text { blood } 48 \mathrm{~h} \\
\text { postoperatively, } \\
\text { decrease in } \\
\text { haemoglobin levels } \\
12 \text { h postoperatively, } \\
\text { amount of blood } \\
\text { transfused }\end{array}$ & $\begin{array}{l}\text { Drained blood }(\mathrm{p}=0.05) \text {, } \\
\text { haemoglobin drop }(\mathrm{p}=0.05) \text { and } \\
\text { transfused units were lower in } \\
\text { the TXA group compared with } \\
\text { controls }\end{array}$ \\
\hline $\begin{array}{l}\text { Carvalho et } \\
\text { al }^{31}\end{array}$ & 2015 & 125 & $\begin{array}{l}\text { IA } 1.5 \text { and } 3.0 \mathrm{~g} \text { in } \\
\text { povidone-iodine } \\
\text { solution }\end{array}$ & $\begin{array}{l}\text { Mean postoperative } \\
\text { haemoglobin levels, } \\
\text { blood loss, safety }\end{array}$ & $\begin{array}{l}\text { Higher mean haemoglobin level } \\
(\mathrm{p}=0.001, \mathrm{p}=0.003) \text { and lower } \\
\text { blood loss }(\mathrm{p}=0.07, \mathrm{p}=0.09) \text { in } \\
\text { the TXA group compared with } \\
\text { controls }\end{array}$ \\
\hline Lin et $\mathbf{a l}^{32}$ & 2015 & 120 & $\begin{array}{l}\text { Combined versus I } \\
\text { A versusplacebo } \\
\text { drop; total drain } \\
\text { amount }\end{array}$ & $\begin{array}{l}\text { Mean total blood, } \\
\text { transfusion rate; } \\
\text { postoperative } \\
\text { haemoglobin }\end{array}$ & $\begin{array}{l}\text { Combining preoperative IV } \\
\text { injection and topical } \\
\text { administration of TXA can } \\
\text { effectively reduce blood loss } \\
(\mathrm{p}=0.001) \text {, total drain amount } \\
(\mathrm{p}=0.001) \text { and transfusion rate } \\
(\mathrm{p}=0.009) \text {. }\end{array}$ \\
\hline
\end{tabular}




\begin{tabular}{|c|c|c|c|c|c|}
\hline Author & Year & $\begin{array}{l}\text { Number } \\
\text { of } \\
\text { patients }\end{array}$ & Protocol & $\begin{array}{l}\text { Outcomes } \\
\text { evaluated }\end{array}$ & Results \\
\hline $\begin{array}{l}\text { Gomez- } \\
\text { Barrena et al }^{33}\end{array}$ & 2014 & 78 & $\begin{array}{l}\text { IA } 3 \mathrm{~g} \text { in } 100 \mathrm{ml} \\
\text { saline and two } 15 \\
\mathrm{mg} / \mathrm{kg} \text { IV doses } \\
\text { (before tourniquet } \\
\text { release and after } \\
\text { three hours) }\end{array}$ & $\begin{array}{l}\text { Drain blood loss at } \\
24 \text { and } 48 \text { hours, } \\
\text { transfusion rate }\end{array}$ & $\begin{array}{l}\text { Topical administration is as } \\
\text { effective and safe as IV } \\
\text { administration, with no } \\
\text { differences in blood loss at } 24 \mathrm{~h} \\
(\mathrm{p}=0.948) \text {, blood loss at } 48 \mathrm{~h} \\
(\mathrm{p}=0.837) \text { or transfusion rate } \\
(0 \% \text { in both groups) }\end{array}$ \\
\hline Yang et $\mathbf{a l}^{34}$ & 2015 & 80 & $\begin{array}{l}\text { IA } 500 \mathrm{mg} \text { in } 20 \mathrm{ml} \\
\text { saline }\end{array}$ & $\begin{array}{l}\text { Blood loss, } \\
\text { transfusion rate, } \\
\text { safety }\end{array}$ & $\begin{array}{l}\text { Less total blood loss and lower } \\
\text { transfusion rate in the TXA } \\
\text { group compared with controls } \\
(\mathrm{p}=0.05)\end{array}$ \\
\hline Huang et $\mathbf{a l}^{35}$ & 2014 & 184 & $\begin{array}{l}\text { IV } 3 \\
\mathrm{~g} \text { versuscombined } \\
\text { IV } 1.5 \mathrm{~g}+\text { topical } \\
1.5 \mathrm{~g}\end{array}$ & $\begin{array}{l}\text { Transfusion rate, } \\
\text { total blood loss, } \\
\text { safety }\end{array}$ & $\begin{array}{l}\text { Combined administration } \\
\text { obtains smaller maximum } \\
\text { decline of haemoglobin } \\
\mathrm{p}=0.031) \text {, smaller drain volume } \\
(\mathrm{p}=0.011) \text {, less postoperative } \\
\text { knee pain, less knee swelling, } \\
\text { shorter length of hospital stays. } \\
\text { No differences in transfusion } \\
\text { rate }\end{array}$ \\
\hline Soni et al $^{36}$ & 2014 & 40 & $\begin{array}{l}3 \text { IV } \\
\text { doses versus topical }\end{array}$ & $\begin{array}{l}\text { Blood loss, blood in } \\
\text { drain }\end{array}$ & $\begin{array}{l}\text { No differences in haemoglobin } \\
\text { drop }(p=0.38) \text { and blood in } \\
\text { drain }(p=0.48)\end{array}$ \\
\hline Patel et $\mathbf{a l}^{37}$ & 2014 & 89 & $\begin{array}{l}\text { IV } 10 \mathrm{mg} / \mathrm{kg} \text { versus } \\
\text { topical } 2 \mathrm{~g}\end{array}$ & $\begin{array}{l}\text { Hemoglobin level, } \\
\text { total drain output, } \\
\text { transfusion rate }\end{array}$ & $\begin{array}{l}\text { Systemic and topical TXA } \\
\text { administration found to be } \\
\text { equally effective as in terms of } \\
\text { haemoglobin drop }(\mathrm{p}=0.108) \text {, } \\
\text { transfusion rate }(\mathrm{p}=0.342) \text {, total } \\
\text { drain output ( } \mathrm{p}=0.339) \text { and } \\
\text { safety }\end{array}$ \\
\hline $\begin{array}{l}\text { Sarzaeem et } \\
\text { al }^{38}\end{array}$ & & 200 & $\begin{array}{l}\text { IV } 1500 \\
\text { mg versus IA } 3 \mathrm{~g} \text { in } \\
100 \mathrm{cc} \\
\text { saline versus } 1.5 \mathrm{~g} \\
\text { injected through the } \\
\text { drain versus } \\
\text { placebo }\end{array}$ & $\begin{array}{l}\text { Hemoglobin drop, } \\
\text { blood in drain, } \\
\text { transfusion rate }\end{array}$ & $\begin{array}{l}\text { All administrations showed } \\
\text { smaller haemoglobin drop } \\
\text { versus controls ( } \mathrm{p}=0.05) ; \mathrm{IV} \\
\text { administration is more effective } \\
\text { in reducing haemoglobin drop } \\
\mathrm{p}=0.05 \text { ) and transfused units } \\
\text { ( } \mathrm{p}=0.031 \text { ) compared with other } \\
\text { groups. Joint irrigation is better } \\
\text { than drug administration } \\
\text { through the drain for obtaining } \\
\text { haemoglobin drop ( } \mathrm{p}=0.001)\end{array}$ \\
\hline $\begin{array}{l}\text { Sa- } \\
\text { Ngasoongsong } \\
\text { et al }{ }^{39}\end{array}$ & 2013 & 135 & $\begin{array}{l}\text { IA } 250 \\
\text { mg versus IA } 500 \\
\text { mg (both injected } \\
\text { through the drain } \\
\text { with a } 2 \text { h drain } \\
\text { clamping) }\end{array}$ & $\begin{array}{l}\text { Hemoglobin drop, } \\
\text { transfusion rate, } \\
\text { safety }\end{array}$ & $\begin{array}{l}\text { The two protocols were equally } \\
\text { effective in reducing total blood } \\
\text { loss }(\mathrm{p}=0.001) \text { and transfusion } \\
\text { rate }(\mathrm{p}=0.05)\end{array}$ \\
\hline $\begin{array}{l}\text { Alshryda et } \\
\text { al }^{40}\end{array}$ & 2013 & 157 & Topical & $\begin{array}{l}\text { Blood transfusion } \\
\text { rate, drain blood } \\
\text { loss, haemoglobin } \\
\text { drop, generic quality } \\
\text { of life (EuroQol), } \\
\text { length of stay, cost, } \\
\text { safety }\end{array}$ & $\begin{array}{l}\text { Topical TXA administration is } \\
\text { effective in reducing transfusion } \\
\text { rate }(p=0.001) \text {, blood loss } \\
(p=0.0003) \text { and length of stay } \\
(p=0.041)\end{array}$ \\
\hline
\end{tabular}




\begin{tabular}{|c|c|c|c|c|c|}
\hline Author & Year & $\begin{array}{l}\text { Number } \\
\text { of } \\
\text { patients }\end{array}$ & Protocol & $\begin{array}{l}\text { Outcomes } \\
\text { evaluated }\end{array}$ & Results \\
\hline $\begin{array}{l}\text { Georgiadis et } \\
\text { al }^{41}\end{array}$ & 2013 & 101 & $\begin{array}{l}\text { IA } 2 \mathrm{~g} \text { in } 75 \text { saline } \\
\text { versus placebo }\end{array}$ & $\begin{array}{l}\text { Blood loss, } \\
\text { transfusion rate, } \\
\text { safety }\end{array}$ & $\begin{array}{l}\text { Smaller haemoglobin loss and } \\
\text { calculated blood loss in TXA } \\
\text { group }(\mathrm{p}=0.001) \text {; differences } \\
\text { were not significant for } \\
\text { transfusion rate }\end{array}$ \\
\hline Lee et $\mathrm{al}^{42}$ & 2013 & 72 & $\begin{array}{l}\text { IV in patients } \\
\text { undergoing } \\
\text { prophylaxis with } \\
\text { factor Xa inhibitor }\end{array}$ & $\begin{array}{l}\text { Blood loss, } \\
\text { transfusion rate, } \\
\text { blood in drain, safety }\end{array}$ & $\begin{array}{l}\text { The treatment group showed } \\
\text { reduced transfusion rate } \\
(\mathrm{p}=0.007) \text { and blood in drain } \\
(\mathrm{p}=0.001) \text {. No differences were } \\
\text { detectable regarding } \\
\text { haemoglobin drop. There was } \\
\text { no interaction with factor Xa } \\
\text { inhibitor }\end{array}$ \\
\hline Seo et $\mathrm{al}^{43}$ & 2013 & 150 & $\begin{array}{l}\text { IV } 1.5 \mathrm{~g} \text { in } 100 \mathrm{cc} \\
\text { saline versus IA } 1.5 \\
\mathrm{~g} \text { in } 100 \mathrm{cc} \text { saline }\end{array}$ & $\begin{array}{l}\text { Blood loss, } \\
\text { transfusion rate }\end{array}$ & $\begin{array}{l}\text { Both TXA groups showed } \\
\text { significantly reduced amount of } \\
\text { blood loss }(\mathrm{p}=0.001) \text { and } \\
\text { transfusion rate }(\mathrm{p}=0.001) \\
\text { compared with the placebo } \\
\text { group. IA administration seems } \\
\text { to be more effective than } \\
\text { systemic administration }\end{array}$ \\
\hline $\begin{array}{l}\text { Chareancholv } \\
\text { anich et } \mathbf{a l}^{44}\end{array}$ & 2012 & 240 & $\begin{array}{l}\text { IV } 10 \mathrm{mg} / \mathrm{kg} 20 \\
\text { min before inflating } \\
\text { the tourniquet, } \\
\text { repeated } 3 \text { hours } \\
\text { after surgery }+ \\
1500 \mathrm{mg} \text { /day of } \\
\text { oral TXA for } 5 \\
\text { days + drainage } \\
\text { clamping }\end{array}$ & $\begin{array}{l}\text { Blood in drain at } 48 \\
\text { hours } \\
\text { postoperatively, hem } \\
\text { oglobin drop, } \\
\text { transfusion rate }\end{array}$ & $\begin{array}{l}\text { TXA is effective in reducing } \\
\text { volumes of drained blood and } \\
\text { amount of blood transfusion } \\
\text { compared with placebo } \\
(\mathrm{p}=0.005) \text { Drain clamping } \\
\text { combined with TXA } \\
\text { administration is more effective } \\
\text { than using TXA or drain } \\
\text { clamping alone as regards } \\
\text { haemoglobin levels }(\mathrm{p}=0.001) \\
\text { and transfusion rate }(\mathrm{p}=0.05)\end{array}$ \\
\hline Lin et $\mathbf{a l}^{45}$ & 2012 & 151 & $\begin{array}{l}\text { IV } 1 \text { dose of } 10 \\
\mathrm{mg} / \mathrm{kg} \text { versus IV } 2 \\
\text { doses of } \\
10 \mathrm{mg} / \mathrm{kg} \text { versusplac } \\
\text { ebo }\end{array}$ & $\begin{array}{l}\text { Hemoglobin drop, } \\
\text { transfusion rate }\end{array}$ & $\begin{array}{l}\text { A single dose is effective in } \\
\text { reducing blood loss }(\mathrm{p}=0.0001) \\
\text { and transfusion rate }(\mathrm{p}=0.006) \text {. } \\
\text { No differences between single } \\
\text { and double dose administration } \\
\text { (blood loss } \mathrm{p}=0.148 \text {; transfusion } \\
\text { rate } \mathrm{p}=0.672 \text { ) }\end{array}$ \\
\hline Roy et al ${ }^{46}$ & 2012 & 50 & $\begin{array}{l}\text { IA } 500 \mathrm{mg} / 5 \mathrm{ml} \\
\text { saline (through the } \\
\text { drain after wound } \\
\text { closure) }\end{array}$ & $\begin{array}{l}\text { Blood loss, blood in } \\
\text { drain, transfusion } \\
\text { rate }\end{array}$ & $\begin{array}{l}\text { IA TXA administration is } \\
\text { effective in reducing both } \\
\text { haemoglobin drop }(\mathrm{p}=0.05) \text { and } \\
\text { total drain collection at } 48 \text { hours } \\
(\mathrm{p}=0.001)\end{array}$ \\
\hline Lin et $\mathbf{a l}^{47}$ & 2011 & 100 & IV $10 \mathrm{mg} / \mathrm{kg}$ & $\begin{array}{l}\text { Blood loss, } \\
\text { transfusion rate }\end{array}$ & $\begin{array}{l}\text { Effective in reducing both total } \\
\text { blood loss }(\mathrm{p}=0.001) \text { and hidden } \\
\text { blood loss }(\mathrm{p}=0.01)\end{array}$ \\
\hline Ishida et al $^{48}$ & 2011 & 100 & $\begin{array}{l}\text { IA } 2 \mathrm{~g} / 20 \mathrm{ml} \text { saline } \\
\text { (through the drain } \\
\text { after wound } \\
\text { closure) }\end{array}$ & $\begin{array}{l}\text { Blood loss, blood in } \\
\text { drain, transfusion } \\
\text { rate, leg diameter }\end{array}$ & $\begin{array}{l}\text { Decreased blood loss }(\mathrm{p}=0.01) \\
\text { and less knee joint swelling } \\
\text { (circumference at the superior } \\
\text { patellar border) in TXA } \\
\text { group versus controls } \mathrm{p}=0.05\end{array}$ \\
\hline
\end{tabular}

Abbreviations: TXA = tranexamic acid; TKA= total knee arthroplasty; IV= intravenous; IA= intra-articular; Hb= haemoglobin.

Although, some researchers reported a potential increased risk of thrombotic events and some cases of allergic reaction, this study however did not come across any of these complications. ${ }^{49}$ Different Authors have studied the efficacy of IA administration, proposing different doses 
and multiple methods of local administration (washing or through the drain). In particular, Georgiadis et al. randomized patients to two groups receiving either $2 \mathrm{~g}$ of TXA in $75 \mathrm{ml}$ of saline or a placebo solution intraoperatively. ${ }^{41}$ The Authors demonstrated a significant reduction of total blood loss in the TXA group, without the potential complications related to IV administration. Patel et al., in a study of 89 patients who underwent a primary TKA, demonstrated that IV administration of $10 \mathrm{mg} / \mathrm{kg}$ of TXA and IA admin-istration of $2 \mathrm{~g}$ TXA were equally effective in reducing blood loss. ${ }^{37}$ Similarly, various recent studies have demon-strated the efficacy of IA TXA administration in reducing blood loss after TKA.

In addition to this, few recent meta-analyses showed no difference between topical and IV TXA administration, even though some authors reported conflicting results.

Very few studies have compared systemic TXA against local TXA. Jain et al. showed better results in terms of mean total blood loss, transfusion rate and haemoglobin drop, using a combined protocol compared to only IV administration. ${ }^{26}$ Similarly, Lin et al., in a study of 120 patients, demonstrated greater reductions in blood loss, haemoglobin drop, total drain amount and transfusion rate using a combined protocol compared to IA administration alone. ${ }^{32}$ Karaaslan et al. evaluated the efficacy of an association of three different methods of TXA administration in bilateral TKA: a bolus dose of $15 \mathrm{mg} / \mathrm{kg}$ $10 \mathrm{~min}$ before the inflation of the tourniquet, followed by IA administration of $3 \mathrm{~g} 10 \mathrm{~min}$ before the deflation of the tourniquet, associated with an IV infusion of $10 \mathrm{mg} / \mathrm{kg} / \mathrm{h}$ for $3 \mathrm{~h}$ following the surgery. The authors concluded that this method of TXA administration was effective in reducing total blood loss in bilateral TKA. ${ }^{30}$ Huang et al. compared the results of IV TXA administration $(3 \mathrm{~g})$ with those of a combined approach (1.5 g IA and $1.5 \mathrm{~g}$ IV). The Authors concluded that the two approaches were similarly effective in reducing transfusion rate and total blood loss, but the combined protocol gave better results in terms of maximum decline of haemoglobin, drainage volume, postoperative knee pain, knee swelling, length of hospital stays and short-term satisfaction. ${ }^{45}$

In view of the established efficacy of TXA in TKA irrespective of the method of administration, we conducted a preliminary retrospective study in 72 patients, divided into IV group and IA + IV group. The aim of this study was to evaluate whether one method of administration was more effective than the other. The results of the study showed marked differences in haemoglobin loss, amount of blood in the drain, and rate of transfusions between the combined protocol and systemic administration alone.

The main limitation in the study is the absence of a control group. That said, the efficacy of TXA, whether administered topically or systemically, has previously been extensively described and is widely accepted. Finally, the power of the study was certainly reduced by the small size of the sample. However, these are preliminary data, and the study will be continued, introducing a randomization procedure, to confirm the significance of the results obtained.

\section{CONCLUSION}

The study inferred that the combination of local and systemic tranexamic acid was superior than systemic administration alone with lower post op blood loss, lower rates of blood transfusion and lower fall in haemoglobin levels with-out any added complications.

Funding: No funding sources Conflict of interest: None declared

Ethical approval: The study was approved by the institutional ethics committee

\section{REFERENCES}

1. Y. Kalairajah,A. J. Cossey,G. M. Verrall,A. J. Spriggins. The Journal of Bone and Joint Surgery. British volumeVol. 87-B, No. 11KneeFree Access Blood loss after total knee replacement. 2005.

2. Risberg B. The response of the fibrinolytic system in trauma. Acta Chir Scand Suppl. 1985;522:245-71.

3. Janssens M, Joris J, David JL, Lemaire R, Lamy M. High-dose aprotinin reduces blood loss in patients undergoing total hip replacement surgery. Anesthesiology. 1994;80:23-9.

4. Kruithof EK, Nicolosa G, Bachmann F. Plasminogen activator inhibitor 1: Development of a radioimmunoassay and observations on its plasma concentration during venous occlusion and after platelet aggregation. Blood. 1987;70:1645-53.

5. Nakahara M, Sakahashi H. Effect of application of a tourniquet on bleeding factors in dogs. J Bone Joint Surg Am. 1967;49:1345-51.

6. Klenerman L, Chakrabarti R, Mackie I, Brozovic M, Stirling Y. Changes in haemostatic system after application of a tourniquet. Lancet. 1977;1:970-2.

7. Petaja J, Myllynen P, Myllyla G, Vahtera E. Fibrinolysis after application of a pneumatic tourniquet. Acta Chir Scand. 1987;153:647-51.

8. Matthew R Bong, Vipul Patel,Eric Chang, Paul S Issack. Risks associated with blood transfusion after total knee arthroplasty. The Journal of Arthroplasty. 2004;19(3):281-7.

9. de Leede-van der Maarl MG, Hilkens P, Bosch F. The epileptogenic effect of tranexamic acid. J Neurol. 1999;246:843.

10. Rossaint R, Bouillon B, Cerny V, Coats TJ, Duranteau J.. Management of bleeding following major trauma: an updated European guideline. Crit Care. 2010;14:R52.

11. McCormack PL. Tranexamic acid: a review of its use in the treatment of hyperfibrinolysis. Drugs. 2012;72:585-617.

12. Bundesamt für Sicherheit im Gesundheitswesen . SmPC Cyklokapron. 2017. 
13. Tian P, Liu W-b, Li Z-j, Xu G-J, Huang Y-T, Ma X1.The efficacy and safety of tranexamic acid in revision total knee arthroplasty: a meta-analysis, BMC Musculoskelet Disord. 2017;18:273.

14. Levine BR, Haughom BD, Belkin MN. Weighted versus uniform dose of tranexamic acid in patients undergoing primary, elective knee arthroplasty: a prospective randomized controlled trial. J Arthroplasty. 2014;29(9Suppl):186-88.

15. Iwai T, Tsuji S, Tomita T. Repeat-dose intravenous tranexamic acid further decreases blood loss in total knee ar-throplasty. Int Orthop. 2013;37:441-45.

16. Maniar RN, Kumar G, Singhi T, Nayak RM, Maniar PR. Most effective regimen of tranexamic acid in knee arthro-plasty: A prospective randomized controlled study in 240 patients. Clin Orthop Relat Res. 2012;470:2605-12.

17. Alshryda S, Sarda P, Sukeik M. Tranexamic acid in total knee replacement: a systematic review and meta-analysis. J Bone Joint Surg Br. 2011;93:1577585.

18. Zhang H, Chen J, Chen F. The effect of tranexamic acid on blood loss and use of blood products in total knee arthroplasty: a meta-analysis. Knee Surg Sports Traumatol Arthrosc. 2012;20:1742-752.

19. Panteli M, Papakostidis C, Dahabreh Z. Topical tranexamic acid in total knee replacement: a systematic review and meta-analysis. Knee. 2013;20:300-09.

20. Wang H, Shen B, Zeng Y. Comparison of topical versus intravenous tranexamic acid in primary total knee arthroplasty: a meta-analysis of randomized controlled and prospective cohort trials. Knee. 2014;21:987-93.

21. Alshryda S, Sukeik M, Sarda P. A systematic review and meta-analysis of the topical administration of tranexamic acid in total hip and knee replacement. Bone Joint J. 2014;96-B:1005-015.

22. Kim TK, Chang CB, Koh IJ. Practical issues for the use of tranexamic acid in total knee arthroplasty: a systematic review. Knee Surg Sports Traumatol Arthrosc. 2014;22:1849-858.

23. Wu Q, Zhang HA, Liu SL. Is tranexamic acid clinically effective and safe to prevent blood loss in total knee arthroplasty? A meta-analysis of 34 randomized controlled trials. Eur J Orthop Surg Traumatol. 2015;25:525-41.

24. Shemshaki H, Nourian SM, Nourian N. One step closer to sparing total blood loss and transfusion rate in total knee arthroplasty: a meta-analysis of different methods of tranexamic acid administration. Arch Orthop Trauma Surg. 2015;135:573-88.

25. Yue C, Pei F, Yang P. Effect of topical tranexamic acid in reducing bleeding and transfusions in TKA. Orthopedics. 2015;38:315-24.

26. Jain NP, Nisthane PP, Shah NA. Combined administration of systemic and topical tranexamic acid for total knee arthroplasty: can it be a better regimen and yet safe? A randomized controlled trial. J Arthroplasty. 2016;31:542-47.
27. Aguilera X, Martínez-Zapata MJ, Hinarejos P. Topical and intravenous tranexamic acid reduce blood loss compared to routine hemostasis in total knee arthroplasty: a multicenter, randomized, controlled trial. Arch Orthop Trauma Surg. 2015;135:1017-025.

28. Shen PF, Hou WL, Chen JB. Effectiveness and safety of tranexamic acid for total knee arthroplasty: a prospective randomized controlled trial. Med Sci Monit. 2015;21:576-81.

29. Hourlier H, Reina N, Fennema P. Single dose intravenous tranexamic acid as effective as continuous infusion in primary total knee arthroplasty: a randomised clinical trial. Arch Orthop Trauma Surg. 2015;135:465-71.

30. Karaaslan F, Karaoğlu S, Mermerkaya MU, et al. Reducing blood loss in simultaneous bilateral total knee arthroplasty: combined intravenous-intraarticular tranexamic acid administration. A prospective randomized controlled trial. Knee. 2015;22:131-135.

31. Carvalho LH, Jr, Frois Temponi E, Machado Soares LF. Bleeding reduction after topical application of tranexamic acid together with Betadine solution in total knee arthroplasty. A randomised controlled study. Orthop Traumatol Surg Res. 2015;101:83-87.

32. Lin SY, Chen $\mathrm{CH}, \mathrm{Fu}$ YC. The efficacy of combined use of intraarticular and intravenous tranexamic acid on reducing blood loss and transfusion rate in total knee arthroplasty. J Arthroplasty. 2015;30:776-80.

33. Gomez-Barrena E, Ortega-Andreu M, PadillaEguiluz NG. Topical intra-articular compared with intravenous tranexamic acid to reduce blood loss in primary total knee replacement: a double-blind, randomized, controlled, noninferiority clinical trial. J Bone Joint Surg Am. 2014;96:1937-944.

34. Yang Y, Lv YM, Ding PJ. The reduction in blood loss with intra-articular injection of tranexamic acid in unilateral total knee arthroplasty without operative drains: a randomized controlled trial. Eur J Orthop Surg Traumatol. 2015;25:135-39.

35. Huang Z, Ma J, Shen B. Combination of intravenous and topical application of tranexamic acid in primary total knee arthroplasty: a prospective randomized controlled trial. J Arthroplasty. 2014;29:2342-346.

36. Soni A, Saini R, Gulati A. Comparison between intravenous and intra-articular regimens of tranexamic acid in reducing blood loss during total knee arthroplasty. J Arthroplasty. 2014;29:1525-527.

37. Patel JN, Spanyer JM, Smith LS. Comparison of intravenous versus topical tranexamic acid in total knee arthroplasty: a prospective randomized study. J Arthroplasty. 2014;29:1528-531.

38. Sarzaeem MM, Razi M, Kazemian G. Comparing efficacy of three methods of tranexamic acid administration in reducing haemoglobin drop following total knee arthroplasty. J Arthroplasty. 2014;29:1521-524.

39. Sa-Ngasoongsong P, Wongsak S, Chanplakorn P. Efficacy of low-dose intra-articular tranexamic acid 
in total knee replacement; a prospective tripleblinded randomized controlled trial. BMC Musculoskelet Disord. 2013;14:340.

40. Alshryda S, Mason J, Vaghela M. Topical (intraarticular) tranexamic acid reduces blood loss and transfusion rates following total knee replacement: a randomized controlled trial (TRANX-K) J Bone Joint Surg Am. 2013;95:1961-968.

41. Georgiadis AG, Muh SJ, Silverton CD. A prospective double-blind placebo controlled trial of topical tranexamic acid in total knee arthroplasty. J Arthroplasty. 2013;28(Suppl 8):78-82.

42. Lee SH, Cho KY, Khurana S. Less blood loss under concomitant administration of tranexamic acid and indirect factor $\mathrm{Xa}$ inhibitor following total knee arthroplasty: a prospective randomized controlled trial. Knee Surg Sports Traumatol Arthrosc. 2013;21:2611-617.

43. Seo JG, Moon YW, Park SH. The comparative efficacies of intra-articular and IV tranexamic acid for reducing blood loss during total knee arthroplasty. Knee Surg Sports Traumatol Arthrosc. 2013;21:1869-74.

44. Chareancholvanich K, Siriwattanasakul P, Narkbunnam R. Temporary clamping of drain combined with tranexamic acid reduce blood loss after total knee arthroplasty: a prospective randomized controlled trial. BMC Musculoskelet Disord. 2012;13:124.
45. Lin PC, Hsu CH, Huang CC. The blood-saving effect of tranexamic acid in minimally invasive total knee replacement: is an additional pre-operative injection effective? J Bone Joint Surg Br. 2012;94:932-36.

46. Roy SP, Tanki UF, Dutta A. Efficacy of intraarticular tranexamic acid in blood loss reduction following primary unilateral total knee arthroplasty. Knee Surg Sports Traumatol Arthrosc. 2012;20:2494-501.

47. Lin PC, Hsu CH, Chen WS. Does tranexamic acid save blood in minimally invasive total knee arthroplasty? Clin Orthop Relat Res. 2011;469:1995002 .

48. Ishida K, Tsumura N, Kitagawa A. Intra-articular injection of tranexamic acid reduces not only blood loss but also knee joint swelling after total knee arthroplasty. Int Orthop. 2011;35:1639-645.

49. A Sundström,H Kieler, L Alfredsson. The risk of venous thromboembolism associated with the use of tranexamic acid and other drugs used to treat menorrhagia: a case-control study using the General Practice Research Database BJOG. An International J Obstetr Gynaecol. 2011;116(1).

Cite this article as: Kamat SG, Dessai R. Retrospective comparative study of IV+ local tranexamic acid versus IV tranexamic acid only in primary total knee replacement. Int J Res Orthop 2021;7:308-18. 